\title{
Kreativität und Formelhaftigkeit in der Realisierung von Komplimenten: Ein deutsch-kamerunischer Vergleich*
}

\author{
Bernard Mulo Farenkia (Yaoundé/Saarbrücken)
}

\begin{abstract}
The large literature on compliments so far reveals that the way these speech acts are uttered depends on languages, countries or cultures. A look at the compliment behaviour of Germans and Americans for instance shows that they are fond of formulaic expressions. The present paper aims at underscoring that most Cameroonians, unlike most Germans, use variable, complex and imaginative structures to express praise or admiration. The study particularly focuses on stylistic and lexical devices.
\end{abstract}

\section{$1 \quad$ Einleitung}

Zahlreiche Studien dokumentieren sprach-, länder- oder kulturspezifische Realisierungsstile von Komplimenten. ${ }^{1}$ Ein Blick auf das Komplimentverhalten im deutschen Sprachraum zum Beispiel zeigt den Rückgriff auf formelhafte bzw. vorgefertigte Formulierungen sowie wertende Lexeme wie Adjektive, Verben u.a. (vgl. Adamzik 1984; Marten-Cleef 1991). Es lässt sich also eine gewisse kommunikative Routine feststellen, die nach Stein (2004: 268)

auf dem Wissen darüber [beruht], welche sprachlichen Mittel und Strukturen in einer Sprachgemeinschaft üblicherweise für welche Aspekte mündlicher Kommunikation verwendet werden können (zuweilen aber auch verwendet werden müssen), und sie besteht in der Fähigkeit, diese sprachlichen Mittel und Strukturen ohne größeren Verbrauch von Planungsressourcen im Zuge der Textherstellung zu reproduzieren.

Manes/Wolfson (1981: 115) ihrerseits hatten in Bezug auf amerikanische Komplimente bereits das fast vollständige Fehlen von Originalität in der Formulierungsweise festgestellt:

One of the most striking features of compliments in American English is their almost total lack of originality. An initial examination of a large corpus reveals a surprising repetitiveness in both the object of the compliments and the lexical items used to describe them. (...) Compliments are, in fact, formulas.

\footnotetext{
* Diese Arbeit stellt Teilergebnisse meines Forschungsprojekts 'Kommunikative Strategien der Beziehungsgestaltung im Kulturvergleich' dar, das von der Alexander von Humboldt Stiftung gefördert und an der Universität des Saarlandes (Saarbrücken) durchgeführt wird.

${ }^{1}$ Vgl. u. a. Manes/Wolfson (1981), Chen (1993), Musry/Wilson (2001), Nixdorf (2002), Probst (2003), http://www.carla.umn.edu/speechacts/bibliography/compliments.html.
} 
Ziel des vorliegenden Beitrags ist es, von diesen Beobachtungen ausgehend und anhand von ausgewählten Realisierungsformen für Komplimente im deutschen und kamerunischen Sprachraum zu zeigen, dass Kameruner, im Gegensatz zu Deutschen, Komplimentanlässe durch den Gebrauch von variantenreichen, komplexen und phantasievollen Strukturen bewältigen. Ich möchte mich hier auf diejenigen Elemente konzentrieren, mit denen der Sprecher versucht, die Aufmerksamkeit des Adressaten derart zu lenken, dass dieser das Kompliment unmissverständlich interpretiert. Unter den zahlreichen Signalen, derer man sich dabei bedienen kann, habe ich lexikalische und stilistische Merkmale deshalb ausgewählt, weil sie am offensichtlichsten zeigen, ob Komplimente in einer Sprachgemeinschaft knapp oder aufwändig, direkt oder indirekt, formelhaft oder kreativ realisiert werden.

Für die empirische Untersuchung zum Vergleich deutscher und kamerunischer Realisierungsstile von Komplimenten wurde ein Korpus ${ }^{2}$ von Daten zusammengestellt, die im Jahre 2001 an der Universität des Saarlandes (Saarbrücken) und 2002 an der Universität von Yaoundé I (Kamerun) im Rahmen einer schriftlichen Befragung erhoben wurden. Insgesamt 163 Studierende (118 in Kamerun und 45 in Deutschland) erhielten die Beschreibung von sieben Situationen und wurden darum gebeten, anzugeben, wie sie die entsprechenden Situationen bewältigen würden, indem sie Komplimente machen (vgl. Anhang). Zusätzlich zur schriftlichen Befragung wurden halbstrukturierte Interviews über das Komplimentverhalten durchgeführt. Im Folgenden werden einige Ergebnisse mit Blick auf die Ausdrucksweisen im stilistischen und lexikalischen Bereich vorgestellt.

\section{Realisierungsstile von Komplimenten im deutsch-kamerunischen Vergleich}

Aus einer umfangreichen Untersuchung zum Komplimentverhalten im deutschen und kamerunischen Sprachraum (Mulo Farenkia 2004) ergab sich, dass die Deutschen häufig auf vorgefertigte Ausdrucksformen rekurrieren, um ihre Intentionen zu vermitteln. Die Kameruner ihrerseits tendieren dazu, kreative, phantasievolle Formen zu erzeugen, um ihren emotionalen Zustand zum Ausdruck zu bringen. Von dieser allgemeinen Feststellung ausgehend möchte ich annehmen, dass sich das Komplimentverhalten im deutschen Sprachraum durch einen formelhaften Realisierungsstil auszeichnet:

Formelhaft sind sprachliche Einheiten, die durch Rekurrenz, d.h. durch häufigen Gebrauch fest geworden sind oder fest werden. Aufgrund der Festigkeit im Gebrauch sind oder werden sie lexikalsiert, d.h. sie sind Bestandteile oder werden zu Bestandteilen des Wortschatzes, so dass sie von den Sprachteilhabern als fertige komplexe Einheiten reproduziert werden (Stein 1995: 57).

Dagegen ist eine grenzenlose Kreativität des Sprechers (Metaphernbildungen, Vergleiche, Neologismen, Sprachkreuzungen, Entlehnungen u.a.) für das Komplimentverhalten im kamerunischen Sprachraum kennzeichnend.

Es wird nun gezeigt, wie sich die Formelhaftigkeit und die Kreativität in den ausgewählten Bereichen manifestieren. Dass der Äußerung jedes Kompliments das Anliegen des Sprechers

\footnotetext{
2 Dieses Korpus ist Teil einer größeren heterogenen empirischen Materialbasis, die die Grundlage verschiedener Untersuchungen des Verfassers zu Komplimenten im deutschen und kamerunischen Sprachraum bildet.
} 
zu Grunde liegt, seinem Gegenüber ein positives Urteil entgegenzubringen, ist allgemein bekannt. Man sollte demzufolge erwarten, dass entsprechende Äußerungen eine positive Einstellung des Sprechers ausdrücken:

It is obvious that since compliments are expression of positive evaluation, every compliment must include at least one term which carried positive semantic load (Manes/Wolfson 1981: 116).

Indikatoren der positiven Bewertung können lexikalisch-semantischer, syntaktischer, stilistischer oder situativer Natur sein. Sehr oft ergibt sich das Kompliment aus der Kombination von Merkmalen verschiedener Ebenen. Die kamerunischen und deutschen Daten weisen eine große Menge positiv konnotierter Lexeme und stilistischer Mittel auf, die die Interpretation von Äußerungen als Komplimente ermöglichen.

\subsection{Kreativität und Formelhaftigkeit im stilistischen Bereich}

Charakteristisch für das kamerunische Komplimentverhalten ist die Tendenz zum hyperbolischen, aufwändigen und indirekten Realisierungsstil. Kameruner sind es gewohnt, das bewertete Objekt größer darzustellen, als es in Wirklichkeit ist. Die übertreibende Ausdrucksweise lässt sich generell an längeren Gesprächssegmenten erkennen, in denen starke Emotionalität signalisierende Merkmale redundant auftreten. Sehr oft tauchen hyperbolische Komplimente in Form von Metaphern und Vergleichen auf. Diese stilistischen Mittel sind die Folge einer starken Bindung an die kamerunische Kultur und einer expliziten Bezugnahme auf ausländische (vor allem europäische und amerikanische) Kulturträger. Die metaphorischen und vergleichenden Ausdrucksweisen können insofern als kreativ betrachtet werden, als der Sprecher in das kulturelle Kollektivbewusstsein hineinwandert, dort geltende Bilder, Stereotypen, Sprichwörter, Redensarten oder Gemeinplätze, die positive Assoziationen aktivieren, herausnimmt und sie auf den wahrgenommenen Sachverhalt sprachlich transferiert. Sein Ziel dabei ist es, nicht nur ein positives Urteil abzugeben, sondern auch seine Bindung an die lokale und die fremde Kultur sowie seine soziokulturelle Identität zu signalisieren. Diese kreative Diskursstrategie stellt ferner auch einen Appell an den Hörer dar, sich aktiv anzustrengen, um das Gemeinte zu erschließen.

\subsubsection{Metaphern}

Metaphern zum Beispiel bilden ein zentrales Mittel der Realisierung von Komplimenten im kamerunischen Sprachraum. Ihr Gebrauch stützt sich, wie bereits gesagt, auf zwei wichtige Quellen, und zwar Elemente der kamerunischen Sprachen und Kultur und Aspekte fremder (vor allem europäischer) Sprachen und Kulturen, die Positives hervorheben. Zwei Gruppen von metaphorischen Komplimenten sind demnach zu unterscheiden.

Die erste Gruppe besteht aus Metaphern, die auf geltenden positiven Gemeinplätzen, Stereotypen oder Topoi in der kamerunischen Gesellschaft basieren. Einige Beispiele dafür sind: Maman tu es une vielle marmite. Diese Metapher geht von der Annahme aus, dass alte Töpfe die besten Speisen liefern. Wird jemand nun als alter Topf bezeichnet, so wird ihm eine 
bemerkenswerte Kompetenz im Bereich des Kochens zugesprochen. Ferner ist in Kamerun zu einem Gemeinplatz geworden, dass die Mutter bzw. Großmutter besser bzw. am besten kocht. Wird eine Mutter/Schwester/Frau als vielle marmite bezeichnet, so werden ihr Komplimente über ihre kulinarischen Leistungen gemacht. Diese Interpretation gilt nur im Kontext der Speisezubereitung. Ein ähnliches Kompliment ist Tu fais la cuisine/tu prépares comme ma grand-mère. Auf Grund der positiven Einstellung zum Alter im kulinarischen Kontext erweckt das Wort grand-mère positive Assoziationen. Dass das Alter einen positiven Wert innehat, wird in der Metapher Tu es vraiment un vieux renard ausgedrückt. Diese Äußerung wird gebraucht, wenn der Sprecher die Kompetenz, die Reife, die Erfahrung des Angesprochenen lobend hervorheben will. Diese Komplimentform wurde in den Situationen 2 und 3 verwendet, in Kontexten also, wo die Leistung des Adressaten Bewunderung erregt. Ein weiteres metaphorisches Kompliment über die vom Gegenüber angebotene Speise ist: J'ai failli manger mes doigts. Diese Äußerung gilt als elliptische Form einer komplexeren Formulierung: Le repas est/était tellement appétissant que j'ai failli manger mes doigts. Hier hinterlässt die Esskultur der kamerunischen Gesellschaft eine Spur in der Komplimentform. Viele typisch kamerunische Spezialitäten werden mit der Hand gegessen. Der Metapher liegt die alltägliche Szene einer mit der Hand essenden Person zu Grunde. Implizit wird zum Ausdruck gebracht, dass der Sprecher mit so großem Appetit gegessen hat, dass er zwischen seinen eigenen Fingern und dem Essen beinahe keinen Unterschied hat machen können. Diese Metapher wird also gebraucht, um das Köstliche lobend hervorzuheben. ${ }^{3}$

Viele Kameruner gehen von positiven Werten aus, die bestimmte Gegenstände, Eigenschaften u. a. symbolisieren, um sie in ihre Metaphern zu integrieren. Dabei werden sprachlich bestimmte Bilder konstruiert. Jemandem ein Kompliment machen heißt zum Beispiel le mettre en haut. Daraus ist die Metapher Tu es en haut entstanden. Die Adverbialgruppe en haut wird hier als Metapher mit der Bedeutung 'an der Spitze sein' bzw. 'das höchste denkbare/erreichbare Maß erreichen' verwendet. Dadurch wird vermittelt, dass der Adressat auf Grund des wahrgenommenen Sachverhalts eine höhere bzw. beneidenswerte Position in der Gesellschaft innehat. Die Äußerung Tu es en haut kann demzufolge durch 'Du bist im Himmel'/'Du bist mir überlegen'/'Du bist Spitze' wiedergegeben werden. Durch diese Metapher wird also ein Bild erzeugt, das die verschiedenen sozialen Stellungen der Gesprächspartner veranschaulicht: Der eine (der Empfänger des Kompliments) wird höhergestellt, weil er etwas Bestimmtes besitzt, der andere (der Sprecher des Kompliments) setzt sich herab und beneidet seinen Partner, weil er das nicht hat, was den Partner zum Überlegenen macht. Jemanden loben bzw. ihm Komplimente machen heißt auch, ihn für etwas Großartiges halten. Das Großartige wird in der kamerunischen Gesellschaft durch einen großen Baum (Baobab) symbolisiert: Tu es un baobab. Wird ein Gesprächspartner im kamerunischen Kontext als Baobab bezeichnet, so wird er, auf Grund seiner beneidenswerten Leistung zum Beispiel, als eine sozial sehr wichtige Person dargestellt. Das Aussehen einer Frau wird sehr oft mit Bezug auf seine Wirkung auf den Adressaten lobend thematisiert. Dabei kann sich der Sprecher Äußerungen bedienen, die als anzüglich interpretiert werden könnten. Um Tabubruch zu

\footnotetext{
${ }^{3}$ Es sei hinzugefügt, dass der Genuss zum Beispiel auch daran festgemacht werden kann, ob und wie sich der Gast die Finger beim Essen leckt.
} 
vermeiden haben Kameruner metaphorische Komplimente entwickelt wie Tu es une grenade; Tu es une bombe; Tu es canon; Tu es bien emballée. Diese Äußerungen thematisieren implizit die erotische bzw. Bombenwirkung, die das Aussehen der angesprochenen Frau auf den Sprecher hat.

Mit den Metaphern wird auch zum Ausdruck gebracht, dass der Komplimentempfänger ein außerordentliches Individuum ist: Tu n'es pas n'importe qui; Un grand n'est pas un petit. Diese metaphorischen Komplimente zeigen, dass Kameruner die multilinguale und multikulturelle Situation nutzen, um hybride Formen zu kreieren, die von Nicht-Kamerunern schwer verstanden werden können. Einige metaphorische Komplimente entstammen aber auch kamerunischen Sprachen, in denen die Bewunderung eines Gesprächspartners einer Huldigung gleichkommt: Je t'arrête aux pieds/Je te prend par les pieds. Diese Metapher, die eine wörtliche Übernahme einer entsprechenden Komplimentformel aus der Beti-Sprache darstellt, präsentiert das Bild eines Sprechers, der durch tiefe Verbeugung die Wertanerkennung des Adressaten ausdrückt. In anderen Sprachen und Regionen heißt es, dass ein Adressat 'jemand' ist, wenn er etwas Großartiges geleistet hat. Daraus ergibt sich das metaphorische Kompliment Tu es vraiment quelqu'un. In vielen Situationen können Wörter wie homme, femme usw. als Symbole positiver Werte in Metaphern wie Tu es vraiment un homme, Tu es une femme verwendet werden.

Nicht alle Metaphern stützen sich ausschließlich auf kamerunische Realitäten. Der Komplimentspender kann, auf Grund allgemein geltender positiver Einstellungen zum Exotischen, den Komplimentempfänger mit Figuren europäischer oder amerikanischer Herkunft metaphorisch identifizieren, die einen hohen Imponierwert oder ein starkes Prestigegefühl in der kamerunischen Gesellschaft entwickeln. Dabei geht es sehr oft um international etablierte Persönlichkeiten, Filmstars, Popstars, Models, Gegenstände u.a. Man vergleiche drei Beispiele aus dem Korpus: Vous avez une maison blanche; Tu es une Naomie Campbell; Avec ta coiffure tu es ma Lady Diana. Mit diesen Äußerungen bewertet der Sprechende das Aussehen seiner Partnerin positiv, wobei er dieser Eigenschaften des Top-Models (Naomie Campbell) oder der Prinzessin (Diana) zuschreibt. Bei diesen Metaphern werden Personen herangezogen, die Schönheitsideale darstellen. Die Situation 6, in der die Schönheit einer Person den Komplimentgegenstand bildet, stellt in Kamerun einen Bereich dar, wo metaphorische Komplimente besonders häufig auftreten. Der Rekurs auf Formen, die Exotisches einbeziehen, dient vorwiegend dazu, die positiven Bewertungen glaubwürdiger zu machen.

Im Gegensatz zu dieser Vorliebe für indirekte Realisierungsstile im kamernischen Kontext verfahren die Deutschen, den Belegen zufolge, ritualisiert und karg. Das Anliegen, sich so schnell wie möglich verständlich zu machen und Mehrdeutigkeiten zu vermeiden, mag die meisten deutschen Gesprächspartner zur Metapher-Abstinenz in ihrem alltäglichen Komplimentverhalten bewegen. Es muss allerdings darauf aufmerksam gemacht werden, dass durchaus Situationen vorstellbar sind, in denen die Interaktionspartner auf milieu- oder gruppenspezifische metaphorische Komplimente zurückgreifen, um zwischen ihnen bestehende Beziehungsverhältnisse sprachlich zu verarbeiten. 


\subsubsection{Vergleiche, Wiederholungen und Code-switching}

Im kamerunischen Korpus tauchen zwei Formen von Komplimenten mittels Vergleichen auf. Die erste Form besteht darin, dass der Sprecher das bewertete Objekt anderen gegenüberstellt und zugleich bekannt gibt, dass das im Mittelpunkt stehende Objekt weitaus besser ist. Dabei bedient er sich Indikatoren wie plus, meilleur, sans pareil, aussi + Adjektiv + que, la/le plus + Adjektiv, ne jamais, défier. Mit diesen Merkmalen signalisiert der das Kompliment Aussprechende, dass die positiv bewerteten Sachverhalte, die bezeichneten Leistungen des Partners ihm bisher nicht begegnet sind. Bewertende Vergleiche gelten als Formen der Partnererhöhung, wenn das Vergleichsobjekt positive Assoziationen aktiviert. Man vergleiche folgende Belege: Ce plat a défié tous les autres; Je n'ai jamais vu une femme aussi belle que vous; Papa, tu as joué comme un jeune de vingt ans; Je n'ai jamais vu un chef aussi talentueux que vous.

Die zweite Realisierungsweise mittels wertenden Vergleichen besteht darin, dass der Sprecher seine Komplimente realisiert, indem er - wie bei den deutschen Komplimenten - die thematisierten Sachverhalte idealisiert, d.h. sie mit paradigmatischen Objekten identifiziert. Die Referenzpunkte sind in der Regel weltweit oder lokal berühmte Persönlichkeiten in unterschiedlichen Bereichen (Politik, Sport, Wissenschaft, Kultur u.a.), mit positiver Konnotation versehenene Tiere (lion(ne), renard u.a.) oder beneidenswerte Gegenstände (rose, or), die auf das zu Beschreibende übertragen werden. Die am häufigsten gebrauchten sprachlichen Mittel dabei sind comme, ressembler à, de. Einige Beispiele dazu: Oh lala! Tu es habillée comme un mannequin; Tu es vraiment comme une princesse; Tu as une coiffure de star; Tu ressembles à un Dieu grec; Tu ressembles á Eddy Murphy avec ta coiffure; Tu brilles comme l'or; Tu brilles comme la lune; Tu es vraiment comme une rose.

In den deutschen Belegen wurde eine geringe Zahl von wertenden Vergleichen als Ausdrucksformen von Komplimenten verzeichnet: Du spielst so gut wie ein Profi; Dein Haus ist wie ein Palast; Das ist das Beste, das ich je gegessen habe. Einen besonderen Typ wertenden Vergleichs in deutschen Komplimenten stellt der Gebrauch des definiten Artikels in superlativer Funktion dar. Dabei werden die Bewertungsobjekte paradigmatisiert bzw. idealisiert: Das ist die Frisur (des Jahres); Das ist das Haus. Die deutschen Daten weisen einige Belege von hyperbolischen Komplimentäußerungen auf, in denen Intensifikatoren wie super, wahnsinnig u. a. in Kombinationen mit Adjektiven üblich sind: Deine Klamotten sehen super wunderschön aus; Das Haus ist super gut eingerichtet; Das Essen hat wahnsinnig gut geschmeckt.

Weitere Aspekte des Realisierungsstils von Komplimenten stellen (inhaltliche oder wörtliche) Wiederholungen von bewertenden Merkmalen dar: Jeune homme, tu es beau, galant et très bien habillé; Papa c'était bien joué, tu as été impressionnant sur le stade; Merci mon ami. Le repas était merveilleux. Tu ne cesseras de me surprendre. C'est une cuisine de spécialiste; Tu es sublime, génial. Hinzu kommen Merkmale des Code-switching, die einen komplexen Deutungsprozess und eine Einbindung in die multilinguale Situation Kameruns erforderlich machen. Da Elemente des Code-switching nicht konventionalisiert sind, ist es dem Sprecher überlassen, auf beliebige Sprachen zurückzugreifen. Im Allgemeinen können alle Sprachen in 
Kamerun als Ressourcen für das Code-switching dienen. So werden unzählige freie Variationen als Ausdrucksformen von Komplimenten gebraucht. Einige Beispiele aus dem Korpus sind: Tes chaussures sont le higher level; Tu es nyanga ${ }^{4}$; Tu es un nassara ${ }^{5}$. Die Präferenz für Code-switching bei Komplimenten ist für die kamerunischen Jugendlichen charakteristisch. Auch bei deutschen Jugendlichen wurde ein kreativer Umgang mit der Sprache konstatiert: Dein Outfit ist echt geil; Das sieht sauschön aus. Über die bewertende Funktion hinaus dient diese Sprechweise dazu, die Gruppenidentität zu stärken, die Flexibilität in der rituellen Kommunikation und eine gewisse soziokulturelle Orientierung zu signalisieren (vgl. Androutsopoulos 1998: 523ff.).

\subsection{Kreativität und Formelhaftigkeit im lexikalischen Bereich}

\subsubsection{Adjektive}

Charakteristisch für die beiden Sprachgemeinschaften ist eine sehr starke Präferenz für den Einsatz von Adjektiven. Diese sind demzufolge die wichtigsten Indikatoren der positiven Bewertung. Besonders auffällig ist die formale und semantische Vielfalt der Adjektive in kamerunischen Komplimenten. Einige Beispiele dafür sind: Mon petit tu es beau/chaud/élégant aujourd'hui; Mon gars tu es/as new; Vous êtes toujours frais/clair comme ça?; Tu es très fumant aujourd'hui; Je te trouve très clean dans cette tenue; Ton accoutrement est très cool. Der intensive Adjektivgebrauch lässt sich auf die Kreativität und Freiheit der Sprecher zurückführen. Diese manifestiert sich dadurch, dass viele Adjektive der französischen Sprache durch neu gebildete typisch kamerunische Formen ersetzt werden. Für diese Adjektive ist kennzeichnend, dass sie sich direkt auf ganz spezifische Merkmale bzw. Eigenschaften des Angesprochenen (z. B. die Frisur, die Kleidung oder Aspekte der äußeren Erscheinung) beziehen. So werden die Adjektive galant, élégant, chaud, clair, pimpant, sexy, attirant, new, charmant, joli, mignon, pur, rayonnant, frais u.a. eingesetzt, wenn Komplimente über das Aussehen des Gegenübers (Kleidung, Figur, Frisur) realisiert werden. Andere Adjektive wie succulent, appétissant, delicieux u.a. beschreiben ausschließlich die Kochkompetenz der angesprochenen Person bzw. das von ihr angebotene Essen. Die Adjektive excellent, talentueux, génial, doué, extraordinaire, inégalable, formidable erscheinen in Situationen, in denen dem Adressaten gegenüber Bewunderung für erbrachte Leistungen geäußert wird (Situationen 2 und 3). Eine weitere Gruppe enthält eigenschaftsneutrale Adjektive, d.h. Adjektive, die sich auf allerlei Arten von Komplimentgegenständen beziehen: Es handelt sich dabei um Adjektive wie fantastique, merveilleux, formidable, incroyable, extraordinaire, magnifique, génial u.a. Der Gebrauch von Adjektiven in Komplimenten zeigt eine Vorliebe für Lexeme, die eine sehr starke emotionale Beteiligung des Sprechers verbalisieren. Bei diesen Lexemen geht es um Adjektive aus dem Französischen oder dem Englischen, die im kamerunischen Sprachalltag ganz andere Konnotationen aktivieren als ihre ursprünglichen Bedeutungen. Zu dieser Kategorie zählen vor allem Anglizismen (z. B. cool,

\footnotetext{
${ }^{4}$ Das Wort nyanga stammt aus der Duala- bzw. Ewondo-Sprache und bedeutet 'schön' bzw. 'Schönheit'.

${ }^{5}$ Nassara ist eine Entlehnung aus der Fufulde-Sprache im Norden Kameruns. Dieses Wort bedeutet 'Europäer', 'Weißer' oder einfach 'zivilisierte/moderne Person'.
} 
new, clean, sharp), Neologismen (chaud, frais, pur, clair, mortel, fumant u.a.). Man vergleiche Beispiele wie Tu es chaud, tu es frais, tu es sharp, tu es clair, tu es clean. Diese Belege entstammen Kontexten, in denen das Aussehen (Kleidung, Frisur usw.) im Mittelpunkt steht. Demzufolge lassen sie sich nicht wörtlich in andere Sprachen übersetzen: Wenn man seinem Gesprächspartner zum Beispiel Tu es chaud sagt, so will man nicht auf seine (körperliche) Wärme hinweisen. Man möchte ihm vielmehr mitteilen, dass sein Aussehen bewundernswert ist. Ähnlich gilt es für Äußerungen wie Ton habillement est mortel; Tu as un look fumant, in denen die Adjektive mortel und fumant auf eine positive Einstellung des Sprechers dem Kleid oder der Frisur des Gesprächspartners gegenüber hinweisen. Mortel sollte also nicht im Sinne von 'tödlich' interpretiert werden, sondern als die Superlativform von 'schön/schick'. Lexeme wie chaud, frais, clair u.a. kann man insofern als Kamerunismen bezeichnen, als es sich dabei zwar um französische Wörter handelt, denen aber andere Lesearten zugeschrieben worden sind, und die nur im kamerunischen Sprachraum in entsprechenden Situationen als positive Bewertung verstanden werden. Im Allgemeinen sind Kamerunismen wie chaud, frais, clair, pur mit großem Abstand im Korpus am stärksten vertreten und können in den Komplimentäußerungen als Synonyme betrachtet werden. Kamerunismen ersetzen in vielen Situationen Standardadjektive wie beau, joli, élégant, die sich großer Beliebtheit in der oberen Schicht erfreuen. Die Äußerungen Tu es chaud; Tu es frais; Tu es cool; Tu es clair/pur lassen sich ins Deutsche mit 'Du bist sehr schick gekleidet' übersetzen. Viele Adjektive können im Komparativ oder Superlativ stehen und weisen eine sehr starke positive Aussagekraft auf: Tu es plus chaud aujourd'hui; Je trouve que tu es la plus mignone de la classe. Anglizismen zum Beispiel dienen dazu, einen trivialen Sachverhalt besonders hervorzuheben (vgl. Androutsopoulos 1998: 578). Diese Adjektive wurden also vorwiegend in den Situationen 1, 5 und 6 gebraucht und sind im Jugendmilieu sehr beliebt. Die Adjektive werden sehr häufig mit intensivierenden Adverbien wie vraiment, carrément, vachement, très, toujours, on ne peut plus, hyper, super u.a. kombiniert. Diese Adverbien drücken sehr oft den höchsten Grad aus: Tu t'es débrouillé vachement bien; Vous jouez agréablement bien; Mon chef, vous êtes particulièrement bien habillé aujourd'hui; Franchement, vous êtes beau; Tu es, on ne peut plus, superbel intéressant; Vous êtes hyper élégant.

Das Repertoire von Adjektiven in deutschen Komplimenten ist im Gegensatz zu kamerunischen Belegen weder groß noch variantenreich. Besonders bevorzugt sind Adjektive wie gut, schön, toll, wunderschön, spitze, klasse, prima, schick, die, wie alle anderen Adjektive, attributiv, prädikativ, im Komparativ oder im Superlativ auftreten können. Erwähnenswert ist, dass sie in vielen Beispielen topikalisiert werden, d.h. die erste Stelle im Satz besetzen und als solche dazu dienen, die Aufmerksamkeit des Gesprächspartners auf die positive Bewertung zu lenken. Diese Lexeme werden in vielen Fällen auch durch Adverbien wie sehr, äußerst, richtig, ganz, absolut, wirklich verstärkt. Man vergleiche einige Beispiele dafür: Du hast heute ein schönes Kleid an; Toll hast du gespielt; Du hast aber einen wirklich tollen Anzug!; Das Essen schmeckt wunderbar/gut; Deine Arbeit ist absolut spitze. Wie bereits gesagt, kommt es im Jugendmilieu oft vor, dass 'neue' Adjektive (z. B. geil) gebraucht werden. 


\subsubsection{Verben und Verbalgruppen}

Einstellungsverben und Verbalgruppen gehören auch zu den Komplimentsignalen, mit denen der Sprecher deutlich macht, ob ihm der im Mittelpunkt stehende Sachverhalt gefällt oder nicht. Man vergleiche folgende kamerunische Beispiele: Ta chemise te convient très bien, papa; Cette tenue te va à merveille; Patron, je vous admire; Votre maison est belle; Je suis vraiment charmé par elle; Tu es/as sapé; Ton habit là me plait; J'aime te voir comme ça; Tu es tiré à quatre épingles; J'aime votre chemise; Personne ne te résistera aujourd'hui; Tu sais préparer!; J'avoue que j'ai vraiment été séduit par votre cuisine; Tu t'y connais vraiment; Ça alors! Tu connais ta chose; Votre beauté me coupe le souffle; J'aimerais vraiment avoir une belle coiffure comme toi/comme la tienne. Manche Verben wie plaire, admirer, aimer, trouver, être charmé par couper le souffle heben den emotionalen Zustand hervor, in den der Komplimentanlass den Sprecher versetzt hat. Mit dem Verb aimer avoir beneidet der Sprecher den Status des Dialogpartners und drückt explizit den Wunsch aus, den bewerteten Gegenstand zu besitzen oder davon zu profitieren. Der Sprecher verwendet, anders ausgedrückt, das Verb aimer avoir, um den Komplimentempfänger bzw. den thematisierten Gegenstand als nachahmenswert oder erstrebenswert darzustellen. Die französische idiomatische Konstruktion être tiré à quatre épingles findet vorwiegend in formellen Situationen Anwendung. Die neologische Verbalgruppe être sapé wird im Jugendmilieu bevorzugt und gilt dabei als Pendant von être tiré à quatres épingles.

Bestimmte wertende Verbalgruppen dienen vorwiegend dazu, die positive Überraschung, die Bewunderung des Sprechers über das Verhalten bzw. die Handlung des Gegenübers zum Ausdruck zu bringen. Diese metakommunikativen Konstruktionen können den eigentlichen Komplimentäußerungen voran- oder nachgestellt werden, um, wie bereits angedeutet, die Glaubwürdigkeit des Kompliments zu unterstreichen. Die meisten kamerunischen Verbalgruppen lassen sich im Deutschen mit 'Ich wusste nicht, dass'; 'Ich bin überrascht, dass/wie' u.a. wiedergeben. Einige Beispiele dazu sind: Je ne te savais pas aussi douéltalentueux au football; Je pensais que le chef ne savait s'occuper que du bureau; Tu ne cesseras de me surprendre/de m'impressionner; Tu m'as toujours caché que tu sais te faire élégant; J'ai du mal à croire que c'est vous que j'ai vu jouer comme ça; J'ignorais que tu avais des talents de cuisinier; J'était vraiment loin d'imaginer que tu es un excellent joueur; Je ne t'ai/vous ai pas reconnu dans cette superbe tenue; Il faut vraiment reconnaître que tu as bien travaillé; Il faut avouer que tu es un bel homme; Je dois vous avouer que vous jouez, très bien. Vous êtes d'une grande habileté. Im deutschen Korpus wurden entsprechende wertende Verbalgruppen nicht verzeichnet.

Im deutschen Korpus werden wertende Verben wie gefallen, schmecken, beneiden, umhauen, beeindruckt sein, finden (in der Bedeutung von 'bewerten' oder 'ansehen als'), gelingen, umhauen gebraucht. Diese Einstellungsverben dienen dazu, den bewerteten Gegenständen positive Eigenschaften zuzuschreiben. Zudem beschreiben sie die Empfindung bzw. den emotionalen Zustand, in den die bewerteten Sachverhalte den Komplimentspender versetzt haben: Deine Frisur gefällt mir; Deine Frisur haut mich um; Ich finde deinen Rock phantastisch; Ich beneide dich um dein Aussehen; Deine Arbeit findet meine vollste Zustimmung; Die 
Arbeit ist dir wirklich gelungen. Prima; Das Essen hat wahnsinnig gut geschmeckt; Ich bin beeindruckt von der Architektur deines Hauses. Die Analyse macht deutlich, dass Kameruner über viel mehr wertende Verben als die Deutschen verfügen, um Komplimente zu formulieren.

\section{Abschließende Bemerkungen}

Die Analysen haben gezeigt, dass Komplimentanlässe den Kamerunern die Gelegenheit bieten, Aspekte ihrer sprachlichen Kreativität zur Schau zu stellen. Dieser Umstand manifestiert sich vorwiegend im Gebrauch von Metaphern, Vergleichen und Neologismen, die sowohl auf Exotisches Bezug nehmen als auch an die kamerunische Sprachen- und soziokulturelle Landschaft anknüpfen. Der Rückgriff auf überschwängliche, variationsreiche und phantasievolle stilistische und lexikalische Mittel dient vor allem dazu, die starke emotionale Beteiligung des Komplimentspenders sowie seine soziale Identität zu signalisieren.

Das deutsche Korpus reflektiert dagegen die Tendenz, Mehrdeutigkeit, Aufwand, Komplexität in der Realisierung von Komplimenten zu vermeiden und demnach auf formelhafte Äußerungen zurückzugreifen:

Die Formelhaftigkeit der Komplimente entlastet die Komplimentproduzenten von Entscheidungsdruck, da sie auf etablierte Komplimentiermuster zurückgreifen können, bei denen die Gefahr, dass das Kompliment misslingt, geringer ist, als bei freien Variationen. Die Komplimentempfänger zumindest scheinen die Formelhaftigkeit keineswegs als Kränkung zu empfinden, die ihrer individuellen Leistung nicht gerecht würde. Formelhafte Komplimente sind daher auch "sichere" Komplimente, da sie für beide, den Produzenten und dem Empfänger, erwartbar und erkennbar sind und dadurch Verhaltenssicherheit und Verlegenheit vermeiden helfen" (Ayaß 1999: 294) ${ }^{6}$

\section{Literaturangaben}

Adamzik, Kirsten (1984): Sprachliches Handeln und sozialer Kontakt. Zur Integration der Kategorie 'Beziehungsaspekt' in eine sprechakttheoretische Beschreibung des Deutschen. Tübingen.

Androutsopoulos, Jannis K. (1998) : Deutsche Jugendsprache. Untersuchungen zu ihren Strukturen und Funktionen. Frankfurt am Main.

Ayaß, Ruth (1999): "Versachlicht oder veraltet: Positive Moralisierungen in alläglichen und institutionellen Kontexten". In: Bergmann, J./Luckmann, Th. (eds.): Kommunikative Konstruktion von Moral. Bd. 2: Von der Moral zu den Moralen. Opladen/Wiesbaden: 289-327. Besch, Elmar (1989): Wiederholung und Variation. Untersuchungen ihrer stilistischen Funktionen in der deutschen Gegenwartssprache. Frankfurt am Main/Bern/New York/ Paris.

\footnotetext{
${ }^{6}$ Vgl. dazu auch Stein (2004: 280), dem zufolge es verschiedene Motive für den Rekurs auf formelhafte Sprache gibt, und zwar Verhaltenssicherheit, Symbolisierung sozialer Identität und Entlastung. Zum dritten Punkt schreibt er: "Der Rekurs auf Formelhaftigkeit stellt ein Verfahren dar zur Erleichterung der Kommunikation und der Formulierungsarbeit, d.h. formelhafte Mittel und Strukturen dienen als bewährte, habitualisierte oder konventionalisierte Lösungen für wiederkehrende Kommunikations- und Formulierungsprobleme" (ebd.).
} 
Manes, Joan/Wolfson, Nessa (1981): "The Compliment Formula". In: Coulmas, F. (ed.): Conversational Routine. Explorations in standardized Communication Situations and Prepatterned Speech. The Hague/Paris/New York: 115-132.

Marten-Cleef, Susanne (1991): Gefühle ausdrücken. Die expressiven Sprechakte. Göppingen.

Mulo Farenkia, Bernard (2004): Komplimentverhalten im deutsch-kamerunischen Vergleich. Habilitationsschrift. Universität des Saarlandes.

Mursy, Ahmad Aly/Wilson, John (2001): "Towards a definition of Egyptian complimenting". Multilingua 20, 2: 133-154.

Nixdorf, Nina (2002): Höflichkeit im Englischen, Deutschen, Russischen. Ein interkultureller Vergleich am Beispiel von Ablehnungen und Komplimenterwiderungen. Marburg.

Probst, Julia (2003): "Ein Kompliment in Ehren ... Aspekte eines 'höflichen' Sprechaktes in mehreren Sprachen". In Baumgarten, N./Böttiger, C./Motz, M./Probst, J. (eds.): Übersetzen, Interkulturelle Kommunikation, Spracherwerb und Sprachvermittlung - das Leben mit mehreren Sprachen. Festschrift für Juliane House zum 60. Geburtstag: 210-225 (= Zeitschrift für Interkulturellen Fremdsprachenunterricht [Online] 8; 2/3) http://www.spz.tu-darmstadt.de/projekt_ejournal/jg-08-2-3/beitrag/Deckblatt1.htm

Stein, Stephan (1995): Formelhafte Sprache. Untersuchungen zu ihren pragmatischen und kognitiven Funktionen im gegenwärtigen Deutsch. Frankfurt am Main.

Stein, Stephan (2004): "Formelhaftigkeit und Routine in mündlicher Kommunikation". In: Steyer, K. (ed.): Wortverbindungen - mehr oder weniger fest. Berlin: 262-288.

Internet-Quelle: http://www.carla.umn.edu/speechacts/bibliography/compliments.html. 


\section{Anhang}

Situation 1: Votre vis-à-vis parait beau dans son habillement: Vous lui en faites des compliments. Comment les formulez-vous?

('Ihr(e) Gesprächspartner(in) sieht in der Kleidung, die er/sie trägt, sehr gut aus. Darüber möchten Sie ihm/ihr Komplimente aussprechen. Wie formulieren Sie sie?')

Situation 2: Vous avez été, lors d'un match de football, impressionné par la technique d'un joueur/d'une joueuse. Quels compliments lui faites-vous?

('Sie sind nach einem Fußballspiel von der Spielweise eines Fußballspielers/einer Fußballspielerin beeindruckt. Sie möchten ihm/ihr Komplimente machen. Wie machen Sie das?')

Situation 3: Une personne a fait un bon travail, exemple bien aménagé le jardin ou saisi un texte à l'ordinateur. Quels compliments lui faites-vous pour son savoir-faire?

('Jemand hat eine gute Arbeit geleistet, zum Beispiel den Garten gut angelegt oder einen Text mit dem Computer gut verarbeitet. Wie formulieren Sie Ihre Komplimente dazu?')

Situation 4: Une personne a aménagé dans sa nouvelle maison. Un jour elle vous invite à la visiter. Vous êtes impressionné par la maison. Quels compliments faites-vous à votre vis-àvis?

('Eine Person hat sich ein neues Haus bauen lassen und lädt Sie ein, das neue Haus zu besichtigen. Sie sind davon sehr beeindruckt und machen Ihrem Gastgeber/Ihrer Gastgeberin Komplimente über das Haus. Wie formulieren Sie sie?')

Situation 5: Une personne s'est fait faire une nouvelle coiffure. Vous vous rendez compte que cette coiffure lui va bien. Quels compliments lui faites-vous?

('Eine Person war beim Friseur, um sich die Haare schneiden zu lassen. Ihre neue Frisur steht ihr sehr gut und Sie möchten ihr ein Kompliment darüber machen. Was sagen Sie?')

Situation 6: Vous vous rendez compte qu'une personne est bien faite, jolie, attirante. Quelles formules utilisez-vous pour lui faire des compliments?

('Sie stellen fest, dass eine Person eine gute Figur hat, hübsch aussieht und attraktiv wirkt. Welche sprachlichen Mittel gebrauchen Sie, um dieser Person Ihre Komplimente auszudrükken?')

Situation 7: Vous avez été invité par une personne à passer un week-end chez elle. Le samedi elle vous offre un repas que vous mangez de bon appétit. Après le repas vous faites des compliments à votre hôte(esse). Comment les formulez-vous?

('Sie sind von jemandem eingeladen worden, bei ihm ein Wochenende zu verbringen. Samstagabend bewirtet er Sie mit einem Menü, das Ihnen sehr gut schmeckt. Nach dem Essen wollen Sie Ihrem Gastgeber/Ihrer Gastgeberin Ihre Komplimente über dieses Essen aussprechen. Wie formulieren Sie sie?') 